\title{
Diagnostic Value of Virtual Bronchoscopic Navigation in the Bronchial Tuberculosis Induced Central Airway Stenosis
}

\author{
Li-Ping Cheng $\cdot$ Ye Gu $\cdot$ Xu-Wei Gui $\cdot$ Yong Fang $\cdot$ Hao Wang $\cdot$
}

Wei Sha

Received: November 11, 2019 / Published online: February 25, 2020

(C) The Author(s) 2020

\section{ABSTRACT}

Introduction: Electronic bronchoscopy is invasive and may cause pain. This study aimed to explore the clinical value of virtual bronchoscopic navigation (VBN) in the diagnosis of benign central airway stenosis (CAS) secondary to tracheobronchial tuberculosis (TBT).

Methods: Sixty-eight patients with benign CAS caused by TBT were recruited between July 2015 and December 2017. The location, length and diameter of stenoses were independently determined by VBN and electronic bronchoscopy (EOB), and the sensitivity and specificity of VBN in identifying stenosis were assessed with $\mathrm{EOB}$ as the gold standard.

Results: In 68 patients with TBT, the overall coincidence between $\mathrm{EOB}$ and $\mathrm{VBN}$ in the

Li-Ping Cheng and Ye Gu contributed equally to this work.

Enhanced Digital Features To view enhanced digital features for this article go to https://doi.org/10.6084/ m9.figshare.11604012.

L.-P. Cheng · X.-W. Gui · Y. Fang · W. Sha $(\bowtie)$ Clinical Center of Tuberculosis, Shanghai Key Laboratory of Tuberculosis, Shanghai Pulmonary Hospital, Tongji University, Shanghai, China e-mail: shfksw@tongji.edu.cn

Y. Gu $\cdot$ H. Wang $(\square)$

Department of Endoscopy, Shanghai Pulmonary

Hospital, Tongji University, Shanghai, China

e-mail: wanghao2138@126.com identification of stenosis was $100 \%$. A total of 188 sites were selected from the central airway, and the stenosis was graded into $0 \%, \leq 25 \%$, $26-50 \%, \quad 51-75 \%, \quad 76-90 \%$ and $>90 \%$. The sensitivity of VBN in determining the degree of stenosis was $98.45 \%, 100.00 \%, 100.00 \%$, $100.00 \%, 84.62 \%$ and $0.00 \%$, respectively; the specificity was $91.53 \%, 96.07 \%, 97.09 \%$, $97.08 \%, 97.14 \%$ and $97.30 \%$, respectively; the accuracy rate was $96.28 \%, 96.28 \%, 97.34 \%$, $97.34 \%, 96.28 \%$ and $95.7 \%$, respectively. The length of airway stenosis on EOB was divided into $<10 \mathrm{~mm}, \quad 10-30 \mathrm{~mm}, \quad 30-50 \mathrm{~mm}$ and $>50 \mathrm{~mm}$. There was no significant difference in the length of airway stenosis between VBN and EOB $(t=0.083, P=0.936 ; t=1.340$, $P=0.199 ; \quad t=1.297, \quad P=0.216 ; \quad t=2.186$, $P=0.081)$. In three patients who received stent placement, VBN was able to accurately assess the postoperative expansion.

Conclusion: VBN is helpful for the diagnosis of TBT-induced CBS and may provide important information on the location, length, diameter and cross-sectional area of stenosis for further EOB examination and interventional therapy. VBN is recommended for patients with TBT and those with contradictions to bronchoscopy, as well as for regular follow-up of stable TBT, because it reduces the incidence of injury, avoids repeat operations and shortens treatment time. 
Keywords: Benign central airway stenosis; Electronic bronchoscopy; Tracheobronchial tuberculosis; Virtual bronchoscopic navigation

\section{Key Summary Points}

VBN is helpful for the diagnosis of TBT induced CBS.

VBN may provide important information on the location, morphology, and extent and degree of stenosis for further EOB examination and interventional therapy.

VBN is recommended for patients with TBT.

VBN is recommended for those with contradictions to bronchoscopy and regular follow-up of stable TBT.

\section{INTRODUCTION}

In China, the main cause of benign central airway stenosis (CAS) is tracheobronchial tuberculosis (TBT). It was reported that TBT accounts for about $60 \%$ of benign airway stenosis of any cause [1].

Electronic bronchoscopy (EOB) is an indispensable approach to the diagnosis of TBT. The routine bronchoscopy and treatment in TBT patients may cause pain and cause patients psychologic distress. Virtual bronchoscopic navigation (VBN) is an emerging peripheral guidance technique, which significantly improves the operational efficiency of diagnosing peripheral pulmonary nodules [2]. The American College of Chest Physician (ACCP) guideline recommends the use of endobronchial ultrasonography with a guide sheath (EBUS-GS) and VBN for the bronchoscopic diagnosis of peripheral pulmonary lesions [3]. It has been reported that VBN is as effective as bronchoscopy in the assessment of tracheobronchial stenosis and can obtain accurate information about the stenosis [4-6].
This study aimed to apply DirectPath VBN to reconstruct the three-dimensional bronchus in TBT patients, and the clinical value of VBN in the diagnosis of CAS secondary to TBT was investigated by comparison with EOB.

\section{METHODS}

\section{Subjects}

Patients who were diagnosed with TBT and treated in the Department of Tuberculosis, Shanghai Pulmonary Hospital, were recruited between July 2015 and December 2017. Sixtyeight patients were finally included in this study. There were 17 males and 51 females with mean age of $34.19 \pm 14.22$ years (range 17-69 years) (Fig. 1). This study was approved by the Ethics Committee of Shanghai Pulmonary Hospital. The study was conducted according to the Helsinki Declaration of 1964, as revised in 2013, concerning human and animal rights, and Springer's policy concerning informed consent was followed.

The inclusion criteria were as follows: (1) acid-fast staining after bronchoscopic brush smears or sputum smears were positive and CAS was confirmed on bronchoscopy; (2) patients were aged 16-70 years; (3) the preoperative coagulation detection and platelet counting showed no bleeding tendency and coagulopathy; (4) patients received chest CT at 1-10 days before or after bronchoscopy; (5) the data on follow-up bronchoscopy were complete.

Exclusion criteria were as follows: (1) patients were pregnant or breast-feeding; (2) patients had severe liver, kidney or heart disease or other systemic diseases; (3) patients had contradictions to bronchoscopy; (4) bronchoscopy showed non-central airway stenosis.

\section{Bronchoscopy}

Routine blood tests, electrocardiography, arterial blood gas analysis and detections of prothrombin time and activated prothrombin time were performed before surgery. Local anesthesia was done by nebulization with $2 \%$ lidocaine. 


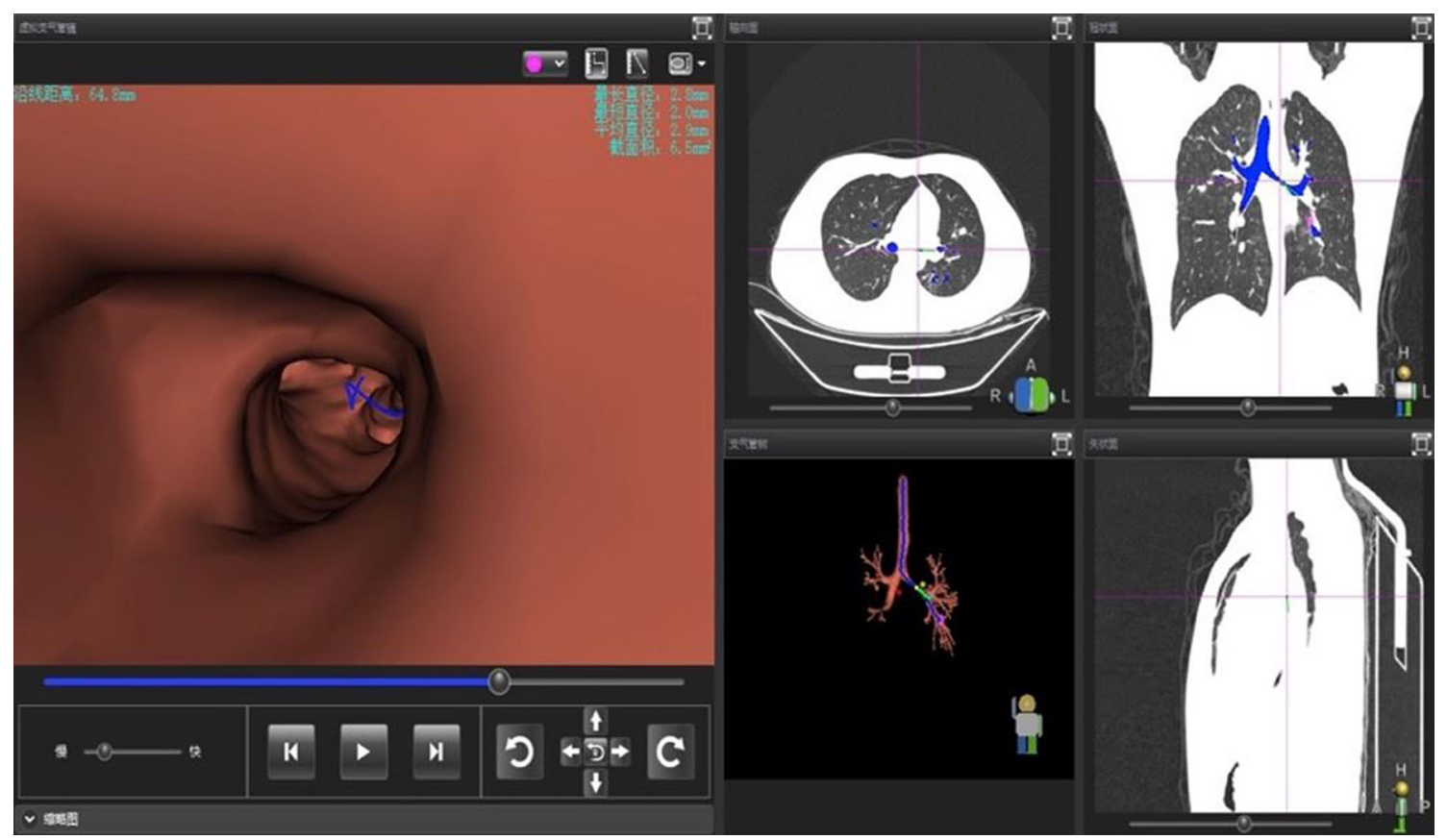

Fig. 1 Full screen on VBN; 4-6 levels of the bronchial tree were displayed; the diameter and cross-sectional area of target bronchia on the VBN and the corresponding

For patients who could not tolerate local anesthesia, general anesthesia was given with propofol, followed by ventilator-assisted ventilation after tracheal intubation, and then bronchoscopy was done. A BFIT260 or BFP260 flexural bronchoscope (outer diameter: $5.9 \mathrm{~mm}$ and $4.0 \mathrm{~mm}$, respectively; Olympus Corp., Japan) was used for examination.

\section{VBN}

All patients received chest thin-section CT (Siemens; LightSpeed VCT64 slice spiral CT; slice thickness: $1 \mathrm{~mm}$ ). The Digital Imaging and Communications in Medicine (DICOM) of CT images was input into the navigation system (DirectPath V1.0, Cybernet Systems; Olympus, Japan), and then VB images of lung lesions were generated. The starting and ending points were set by the operator, and the best path to the target lesion was determined with the software (DirectPath V1.0, Cybernet Systems; Olympus, cross section, coronal section and sagittal section on the chest CT were observable

Japan). This path was used to guide the bronchoscope to the lesion. The location, length, diameter and cross-sectional area of the stenosis were determined during the examination.

\section{Statistical Analysis}

The EOB findings during hospitalization and follow-up were carefully reviewed, and the location, length and diameter of the stenosis were recorded. On the basis of VBN images, the location and length of the stenosis as well as the distal bronchial lumen were assessed by two experienced bronchoscopy operators and then recorded. The findings obtained from two examinations were compared, aiming to assess the practicality of VBN.

Statistical analysis was performed with the Statistical Package for the Social Sciences (SPSS version 19.0, NY, USA). Qualitative data are expressed as number or rate and compared with chi-square test. Quantitative data are expressed 
as mean \pm standard deviation (SD) and were compared with a $t$-test between two groups. $P<0.05$ was considered statistically significant.

\section{RESULTS}

A total of 68 patients were recruited. In 20 patients, the bronchial diameter was $<4 \mathrm{~cm}$, the bronchoscope could not pass through the lesioned bronchia and thus information was incomplete. Of these patients, 59 received initial treatment, and 9 underwent retreatment. The mean course of disease was $2.95 \pm 3.29$ months. Acid-fast staining of the sputum or bronchoscopic brush smears were positive in 55 patients, and pathologic examination after bronchial biopsy on bronchoscopy confirmed tuberculosis in 13 patients.

The findings from VBN and EOB were compared, and results showed the overall coincidence was $100 \%$ in identifying the location of stenosis (Table 1 ).

Then, patients with CAS caused by TBT were further studied. A total of 188 sites were selected from the central airway, including the stenotic airway and normal airway. On EOB, the stenosis was graded as $0 \%$ (no stenosis), $\leq 25 \%$, $26-50 \%, 51-75 \%, 76-90 \%$ and $>90 \%$ [7]. In the VBN, the degree of bronchial stenosis was calculated as the ratio of stenotic bronchial cross-sectional area to normal bronchial crosssectional area and also graded as described above. Data were compared between EOB and VBN, and the sensitivity and specificity of VBN in assessing the degree of bronchial stenosis were determined (Table 2). The sensitivity of VBN was $98.45 \%, 100.00 \%, 100.00 \%, 100.00 \%$, $84.62 \%$ and $0.00 \%$, respectively, and the specificity was 91.53\%, 96.07\%, 97.09\%, 97.08\%, $97.14 \%$ and $97.30 \%$, respectively.

In addition, the length of bronchial stenosis measured by the two techniques was also comparable (Table 3). Of note, the length of bronchial stenosis was not measured in 20 patients because of severe stenosis.

In three patients with severe stenosis, stent placement was done and thereafter EBV was performed to assess the bronchial stenosis. Results showed there were no marked differences in the location and length of stenosis determined by VBN and EOB. VBN was better to precisely determine the stenosis diameter (Table 4).

\section{DISCUSSION}

China has a high prevalence of tuberculosis with about 900,000 new cases of tuberculosis every year; about $10-40 \%$ of active tuberculosis patients have concomitant TBT [8], and about $23.3 \%$ of patients develop severe airway stenosis [9]. Regular bronchoscopy is invasive and may cause pain. EOB still has some operational risks,

Table 1 Location of the stenotic airway determined by two approaches

\begin{tabular}{lccl}
\hline Stenotic airway & EOB $(\boldsymbol{n})$ & VBN $(\boldsymbol{n})$ & Coincidence rate (\%) \\
\hline Left main bronchus & $31(45.6 \%)$ & $31(45.6 \%)$ & 100 \\
Right main bronchus & $12(17.7 \%)$ & $12(17.7 \%)$ & 100 \\
Trachea and left main bronchus & $2(2.9 \%)$ & $2(2.9 \%)$ & 100 \\
Trachea and right main bronchus & $7(10.3 \%)$ & $7(10.3 \%)$ & 100 \\
Trachea, right main bronchus and right middle bronchus & $1(1.5 \%)$ & $1(1.5 \%)$ & 100 \\
Right main bronchus and right middle bronchus & $5(7.3 \%)$ & $5(7.3 \%)$ & 100 \\
Right middle bronchus & $10(14.7 \%)$ & $10(14.7 \%)$ & 100 \\
Total & $68(100 \%)$ & $68(100 \%)$ & 100 \\
\hline
\end{tabular}


Table 2 Degree of airway stenosis determined by two approaches

\begin{tabular}{lcccll}
\hline Degree of stenosis (\%) & EOB $(\boldsymbol{n})$ & \multicolumn{2}{l}{ VBN } & & \\
\cline { 3 - 6 } & & $(\boldsymbol{n})$ & Sensitivity (\%) & Specificity (\%) & Accuracy (\%) \\
\hline 0 & 129 & 127 & 98.45 & 91.53 & 96.28 \\
$\leq 25$ & 10 & 12 & 100.00 & 96.07 & 96.28 \\
$26-50$ & 16 & 16 & 100.00 & 97.09 & 97.34 \\
$51-75$ & 17 & 17 & 100.00 & 97.08 & 97.34 \\
$76-90$ & 13 & 11 & 84.62 & 97.14 & 96.28 \\
$>90$ & 3 & 0 & 0.00 & 97.30 & 95.75 \\
\hline
\end{tabular}

$\mathrm{EOB}$ was used as the gold standard, sensitivity $=$ true positive $/($ true positive + false negative $) \times 100 \%$; specificity $=$ true negative/(true negative + false positive) $\times 100 \%$

Table 3 Length of central airway stenosis

\begin{tabular}{llclcll}
\hline Length of stenosis $(\mathbf{m m})$ & EOB $(\boldsymbol{n})$ & Mean $(\mathbf{m m})$ & VBN $(\boldsymbol{n})$ & Mean $(\mathbf{m m})$ & $\boldsymbol{T}$ & $\boldsymbol{P}$ \\
\hline$<10$ & 11 & $7.7 \pm 1.66$ & 11 & $7.7 \pm 1.69$ & 0.083 & 0.936 \\
$10-30$ & 17 & $20.1 \pm 5.31$ & 17 & $20.3 \pm 5.48$ & 1.340 & 0.199 \\
$30-50$ & 15 & $38.5 \pm 5.05$ & 15 & $38.7 \pm 4.90$ & 1.297 & 0.216 \\
$>50$ & 5 & $57.4 \pm 4.16$ & 5 & $58.1 \pm 4.87$ & 2.186 & 0.081 \\
\hline
\end{tabular}

$t$-test

Table 4 Findings from EOB and VBN after stent placement

\begin{tabular}{|c|c|c|c|c|c|c|}
\hline & \multicolumn{2}{|l|}{ Location of stenosis } & \multicolumn{2}{|c|}{$\begin{array}{l}\text { Length of } \\
\text { stenosis }(\mathbf{c m})\end{array}$} & \multicolumn{2}{|c|}{$\begin{array}{l}\text { Diameter of the most evident } \\
\text { stenosis }(\mathbf{m m})\end{array}$} \\
\hline & $\overline{\text { EOB }}$ & VBN & $\overline{\mathrm{EOB}}$ & VBN & $\overline{\text { EOB }}$ & VBN \\
\hline Case 1 & Trachea, left main bronchus & Trachea, left main bronchus & 11.30 & 11.28 & $4.0 \times 5.0$ & $3.9 \times 5.2$ \\
\hline Case 2 & Trachea & Trachea & 5.36 & 5.40 & $8.0 \times 9.0$ & $8.3 \times 8.9$ \\
\hline Case 3 & Trachea, left main bronchus & Trachea, left main bronchus & 6.30 & 6.31 & $5.0 \times 5.0$ & $4.9 \times 5.2$ \\
\hline
\end{tabular}

In case 1, VBN examination showed the lumen stenosis was significantly improved; VBN still noted evident stenosis in case 2 and case 3 , and then bronchoscopic granulation debridement and balloon dilatation were performed repeatedly

and some patients are unsuitable for EOB because of contradictions.

Virtual bronchoscopy is safe, non-invasive, reproducible and acceptable for most patients. Currently, VBN is mainly used in the diagnosis of peripheral nodules $<2 \mathrm{~cm}$ [10-13]. Regarding CAS, VBN can be used to assess the degree of stenosis and for the preoperative plan and postoperative assessment, but data about the clinical application of VBN are limited and out of date.

As shown in Fig. 1, VBN can mimic the situation of the bronchoscope entering the lesion, display the stenosis and measure the maximum diameter, minimum diameter and cross-sectional area. In addition, the length of stenosis 
corresponding to the site on CT can also be determined on VBN. Our results showed that both VBN and EOB could locate the CAS accurately, and their coincidence rate was $100 \%$. The length of CAS was divided into $<10 \mathrm{~mm}$, $10-30 \mathrm{~mm}, 30-50 \mathrm{~mm}$ and $>50 \mathrm{~mm}$ and then compared between VBN and EOB. Results showed the length of CAS was comparably determined by VBN and EOB. However, the details obtained on VBN are not as clear as those on EOB, especially the mucosal changes, and VBN cannot differentiate the granulation tissues from caseous necrotic tissues and caulking sputum.

Tracheal stenosis is uneven and irregular. Some investigators have investigated measurement of the bronchial diameter with different methods [14-16], and evidence shows that determining the stenotic diameter based on three-dimensional images is feasible [17]. The DirectPath VBN can automatically measure the maximum and minimum diameters as well as the cross-sectional area of the stenosis, can determine the degree and grade of stenosis and may more accurately and precisely display the stenosis, which are helpful for the following treatments. At present, the degree of benign CAS is divided into six grades in China. This classification is mainly determined by the operator, and there is no standard. Begnaud et al. found that there was considerable variation in the visual assessment of central airway obstruction on bronchoscopy [18]. It has been reported that the degree of bronchial stenosis on VBN is closely related to that determined by bronchoscopy $[19,20]$. Multidetector CT virtual bronchoscopy is a reliable noninvasive method that allows accurate grading of tracheobronchial stenosis [21]. Amorico et al. [22] evaluated 25 patients with suspected obstructive tracheobronchial stenosis. They found that VBN was as useful as bronchoscopy in evaluating response to treatment in patients with cancer or severe stenotic lesions. Akhlaghpoor et al. [23] investigated tracheal stenosis secondary to sulfur mustard inhalation and found that the overall sensitivity, specificity and accuracy of VBN in detection of bronchial stenosis were $62.5 \%, 97.8 \%$ and $92.6 \%$, respectively. In the present study, the degree of bronchial stenosis was divided into $0, \leq 25 \%$, $26-50 \%, 51-75 \%, 76-90 \%$ and $>90 \%$, and the degree of bronchial stenosis determined by EOB served as a gold standard. The sensitivity of VBN was $98.45 \%, 100.00 \%, 100.00 \%, 100.00 \%$, $84.62 \%$ and $0.00 \%$, respectively, and the specificity was $91.53 \%, 96.07 \%, 97.09 \%, 97.08 \%$, $97.14 \%$ and $97.30 \%$, respectively, in the determination of the degree of bronchial stenosis. This suggests that the sensitivity of VBN in determining the degree of bronchial stenosis decreases when the CAS is $>75 \%$. In addition, in images the stenotic airway may be different from the true stenotic airway; images from routine chest CT are captured on inspiration, and the dynamic images are neglected. This may be more evident in the VBN of patients with the wall-thickening type bronchial tuberculosis.

In our study, EOB showed the stenosis diameter was $<4 \mathrm{~mm}$ in 20 patients, and thus the bronchoscope could not pass through the stenotic airway (Fig. 2). In 13 patients, the stenotic airway was displayed as a dead end on DirectPath VBN. Eberhardt et al. [24] found that when the diameter of the stenotic airway was $<3 \mathrm{~mm}$ because of inflammation and clogging, the extraction of materials from the bronchial tree with stenosis or blockage failed. This may be the main reason for the reduced sensitivity in the determination of severe CAS on VBN. The additional manual extraction mode of DirectPath VBN can be employed for the extraction of materials from the bronchial tree. If the materials from the bronchial tree are not extracted, the ROI mode of DirectPath VBN may be used to semi-automatically or manually extract the materials from the bronchial tree, and the path can be automatically established. Moreover, the length of stenosis can also be determined, and the airway distal to the stenotic site may be observed (Fig. 3a, b). In clinical practice, this manipulation of the VBN can further guide the subsequent interventional therapy. In the present study, complete occlusion of the left main bronchus was found in one patient; the manual extraction was performed on VBN to determine the length of stenosis and observe the airway distal to the stenotic site; then, the stenotic airway was managed with an 


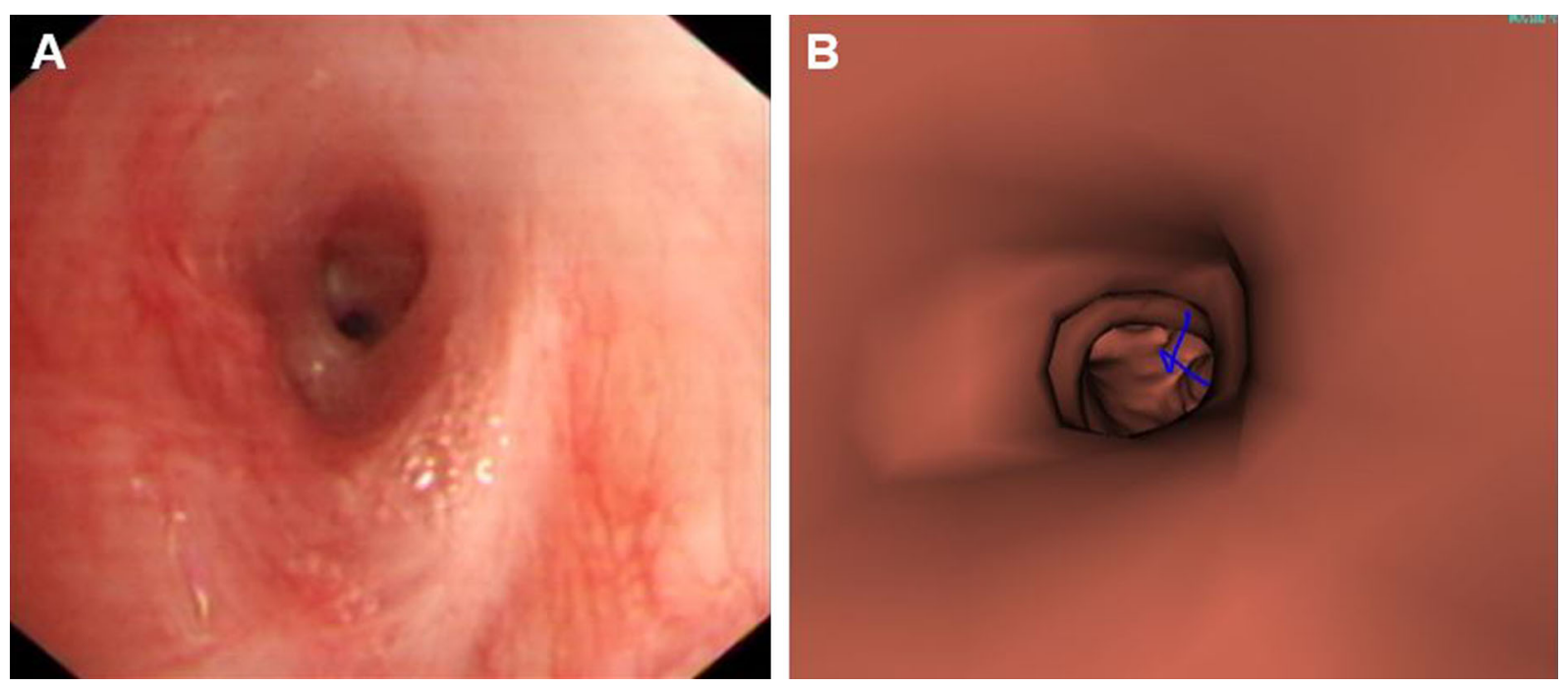

Fig. 2 a Left main bronchus on EOB. The bronchoscope could not pass through the airway because of severe stenosis; $\mathbf{b}$ left main bronchus on VBN. The bronchoscope could pass through the airway
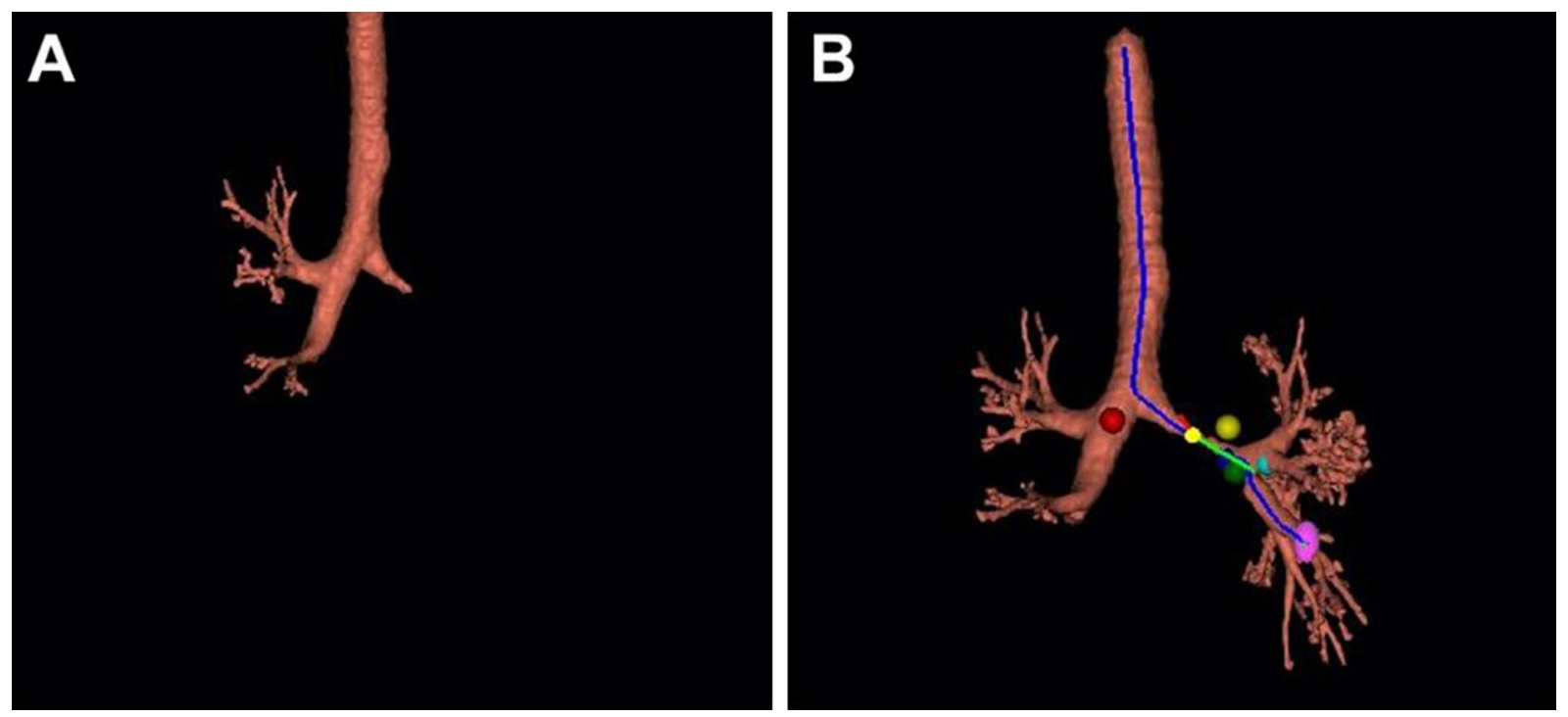

Fig. 3 a Left main bronchial stenosis on VBN and failure to extract materials from the main bronchus. b The airway distal to the stenotic site displayed using the manual extraction mode of VBN

argon knife followed by balloon dilatation, achieving a satisfactory outcome.

The preoperative VBN examination can help understand the disease condition as soon as possible and plan appropriate treatments for EOB, reducing operating time. Asano et al. [25] found preoperative VBN examination could shorten the time required for subsequent endoscopic surgery, and thus they recommended using $\mathrm{VBN}$ for the treatment planning and postoperative evaluation of therapeutic responses in patients with cancer or severe stenosis. In the present study, three patients underwent tracheal stent placement because of severe tracheal stenosis (the filmcovered stent was $6 \mathrm{~cm}$ long in two patients, and the nude stent was $5 \mathrm{~cm}$ in one patient). Results showed the lumen with stent placement 
was still stenotic in two patients; the stenosis was significantly relieved in one patient. Then, these patients received VBN after postoperative chest $\mathrm{CT}$, and the results were compared between VBN and EOB (Table 4). There were no significant differences in the location and length of stenosis between $\mathrm{VBN}$ and $\mathrm{EOB}$, but VBN was better for precisely determining the stenosis diameter. Studies have confirmed that VBN can help understand the airway stenosis and location of stents in patients receiving bronchial stent placement, which reduces unnecessary bronchoscopic manipulations.

Our study shows VBN has clinical value in the diagnosis of CAS secondary to TBT. This technique can be used in patients with contraindications to bronchoscopy to assess the airway stenosis and the airway distal to the stenotic site before surgery, aid the treatment planning and shorten the manipulation. The use of this technique for routine follow-up in patients with stable TBT may reduce suffering and avoid repeat bronchoscopy.

There are limitations of the present study. This was a single-center study, and the sample size was small. VBN's ability to extract materials from the bronchial tree should be further improved in patients with severe CAS, and the airway images obtained may be different from the true situation. Thus, more clinical studies with large sample sizes are needed to confirm the role of VBN in the diagnosis of CAS secondary to TBT. The development of virtual navigation technology will broaden the application of VBN in benign airway stenosis.

\section{CONCLUSION}

VBN is helpful for the diagnosis of TBT-induced CBS and may provide important information on the location, length, diameter and cross section of the stenosis for further EOB examination and interventional therapy. VBN is recommended for patients with TBT and those with contradictions to bronchoscopy as well as for the routine follow-up of stable TBT, which may reduce the patients' suffering, avoid repeat examinations and shorten the time of the manipulation.

\section{ACKNOWLEDGEMENTS}

We thank the participants of the study.

Funding. This study and Rapid Service Fees were supported by the National "13th Five-Year Plan" for the Prevention and Treatment of Infectious Diseases (2018ZX10722302) and Shanghai key clinical specialty construction project (tuberculosis). All authors had full access to all of the data in this study and take complete responsibility for the integrity of the data and accuracy of the data analysis.

Authorship. All authors meet the International Committee of Medical Journal Editors (ICMJE) criteria for authorship for this article, take responsibility for the integrity of the work as a whole, and have given their approval for this version to be published.

Disclosures. Li-Ping Cheng, Ye Gu, Xu-Wei Gui, Yong Fang, Hao Wang and Wei Sha declare that they have no conflict of interest.

Compliance with Ethics Guidelines. This study has been approved by the Ethics Committee of Shanghai Pulmonary Hospital. The study was conducted according to the Helsinki Declaration of 1964, as revised in 2013, concerning human and animal rights, and Springer's policy concerning informed consent has been followed.

Data Availability. The datasets generated during and analyzed during the current study are available from the corresponding author on reasonable request.

Open Access. This article is distributed under the terms of the Creative Commons Attribution-NonCommercial 4.0 International License (http://creativecommons.org/licenses/ by-nc/4.0/), which permits any noncommercial use, distribution, and reproduction in any medium, provided you give appropriate credit to the original author(s) and the source, provide a link to the Creative Commons license, and indicate if changes were made. 
Open Access. This article is licensed under a Creative Commons Attribution-NonCommercial 4.0 International License, which permits any non-commercial use, sharing, adaptation, distribution and reproduction in any medium or format, as long as you give appropriate credit to the original author(s) and the source, provide a link to the Creative Commons licence, and indicate if changes were made. The images or other third party material in this article are included in the article's Creative Commons licence, unless indicated otherwise in a credit line to the material. If material is not included in the article's Creative Commons licence and your intended use is not permitted by statutory regulation or exceeds the permitted use, you will need to obtain permission directly from the copyright holder. To view a copy of this licence, visit http://creativecommons.org/licenses/by$\mathrm{nc} / 4.0 /$.

\section{REFERENCES}

1. Li YQ, Li Q, Bai C, et al. Causes of benign central airway stenoses and the efficacy of interventional treatments through flexible bronchoscopy. Zhonghua Jie He He Hu Xi Za Zhi. 2008;31:364-8.

2. Li S, Yan $\mathrm{W}$, Chen $\mathrm{M}$, Sun $\mathrm{L}, \mathrm{Wu} \mathrm{Q}$, Chen $\mathrm{K}$. Diagnostic utility of virtual bronchoscopic navigation assisted endobronchial ultrasonography with guide sheath for peripheral pulmonary lesions. Zhongguo Fei Ai Za Zhi. 2019;22:125-31.

3. Usuda J. Virtual bronchoscopic navigation (VBN) and electromagnetic navigation system. Kyobu Geka. 2018;71:843-9.

4. Allah MF, Hussein SR, El-Asmar AB, et al. Role of virtual bronchoscopy in the evaluation of bronchial lesions. J Comput Assist Tomogr. 2012;36:94-9.

5. Hoppe H, Dinkel HP, Walder B, von Allmen G, Gugger M, Vock P. Grading airway stenosis down to the segmental level using virtual bronchoscopy. Chest. 2004;125:704-11.

6. Kosucu M, Eroglu A, Besir A, Cansu A. Using Proseal LMA and I-gel for difficult airway management in patient with diffuse tracheal stenosis and pulmonary artery sling. Bratisl Lek Listy. 2013;114: 418-20.
7. Ko Y, Yoo JG, Yi CA, et al. Changes in the flowvolume curve according to the degree of stenosis in patients with unilateral main bronchial stenosis. Clin Exp Otorhinolaryngol. 2015;8:161-6.

8. Kashyap S, Mohapatra PR, Saini V. Endobronchial tuberculosis. Indian J Chest Dis Allied Sci. 2003;45: 247-56.

9. Su Z, Cheng Y, Wu Z, et al. Incidence and predictors of tracheobronchial tuberculosis in pulmonary tuberculosis: a multicentre, large-scale and prospective study in Southern China. Respiration. 2019;97:153-9.

10. Ali MS, Trick W, Mba BI, Mohananey D, Sethi J, Musani AI. Radial endobronchial ultrasound for the diagnosis of peripheral pulmonary lesions: a systematic review and meta-analysis. Respirology. 2017;22:443-53.

11. Diez-Ferrer M, Morales A, Tebe C, et al. Ultrathin bronchoscopy with and without virtual bronchoscopic navigation: influence of segmentation on diagnostic yield. Respiration. 2019;97:252-8.

12. Kato A, Yasuo M, Tokoro Y, et al. Virtual bronchoscopic navigation as an aid to CT-guided transbronchial biopsy improves the diagnostic yield for small peripheral pulmonary lesions. Respirology. 2018;23:1049-54.

13. Yasuo M, Kobayashi $\mathrm{T}$, Hama M, et al. Combination of virtual bronchoscopic navigation with conventional transbronchial needle aspiration in the diagnosis of peribronchial pulmonary lesions located in the middle third of the lungs. Respir Investig. 2016;54:355-63.

14. Gomez-Tamayo JC, Puerta-Guarin JI, Rojas-Camejo $\mathrm{CM}$, Caicedo JP, Calvache JA. Inter-rater and intrarater reliability of the airway diameter measured by sonography. J Ultrasound. 2018;21:35-40.

15. Ma S, Adjavon S, Bouchiha N, et al. Automated measurement of tracheal and main bronchial diameters: a feasibility study. Minerva Anestesiol. 2019;85:1281-8.

16. Vallone N, Pizzo MC, Massaroni C, et al. Design and characterization of a measurement system for monitoring pressure exerted by bronchial blockers: in vitro trials. Conf Proc IEEE Eng Med Biol Soc. 2015;2015:1691-4.

17. Kim D, Son JS, Ko S, Jeong W, Lim H. Measurements of the length and diameter of main bronchi on three-dimensional images in Asian adult patients in comparison with the height of patients. J Cardiothorac Vasc Anesth. 2014;28:890-5. 
18. Begnaud A, Connett JE, Harwood EM, Jantz MA, Mehta HJ. Measuring central airway obstruction. What do bronchoscopists do? Ann Am Thorac Soc. 2015;12:85-90.

19. Mark Z, Bajzik G, Nagy A, Bogner P, Repa I, Strausz J. Comparison of virtual and fiberoptic bronchoscopy in the management of airway stenosis. Pathol Oncol Res. 2008;14:313-9.

20. Shitrit D, Valdsislav P, Grubstein A, Bendayan D, Cohen M, Kramer MR. Accuracy of virtual bronchoscopy for grading tracheobronchial stenosis: correlation with pulmonary function test and fiberoptic bronchoscopy. Chest. 2005;128:3545-50.

21. Hoppe H, Walder B, Sonnenschein M, Vock P, Dinkel HP. Multidetector CT virtual bronchoscopy to grade tracheobronchial stenosis. AJR Am J Roentgenol. 2002;178:1195-200.
22. Amorico MG, Drago A, Vetruccio E, Bollino F, Pizzuti G, Gallo E. Tracheobronchial stenosis: role of virtual endoscopy in diagnosis and follow-up after therapy. Radiol Med. 2006;111:1064-77.

23. Akhlaghpoor S, Ahari AA, Shabestari AA, Ghanei M, Ali HA, Shakiba M. Comparison of virtual bronchoscopy with fiberoptic bronchoscopy findings in patients exposed to sulfur mustard gas. Acta Radiol. 2011;52:1095-100.

24. Eberhardt R, Kahn N, Gompelmann D, Schumann $M$, Heussel CP, Herth FJ. LungPoint-a new approach to peripheral lesions. J Thorac Oncol. 2010;5:1559-63.

25. Asano F. Virtual bronchoscopy. Kyobu Geka. 2007;60:739-44. 